\title{
L-arginine, asymmetric and symmetric dimethylarginine for early outcome prediction in unselected cardiac arrest victims: a prospective cohort study
}

\author{
Beata Csiszar ${ }^{1,2} \cdot$ Zsolt Marton $^{1,2} \cdot$ Janos Riba $^{1} \cdot$ Peter Csecsei $^{3} \cdot$ Lajos Nagy $^{4} \cdot$ Kalman Toth $^{1,2} \cdot$ Robert Halmosi $^{1,2}$. \\ Barbara Sandor $^{1,2} \cdot$ Peter Kenyeres $^{1,2}\left(\mathbb{0} \cdot\right.$ Tihamer Molnar $^{5}$
}

Received: 17 March 2021 / Accepted: 15 May 2021 / Published online: 3 June 2021

(c) The Author(s) 2021

\begin{abstract}
Early prediction of the mortality, neurological outcome is clinically essential after successful cardiopulmonary resuscitation. To find a prognostic marker among unselected cardiac arrest survivors, we aimed to evaluate the alterations of the L-arginine pathway molecules in the early post-resuscitation care. We prospectively enrolled adult patients after successfully resuscitated in- or out-of-hospital cardiac arrest. Blood samples were drawn within 6, 24, and 72 post-cardiac arrest hours to measure asymmetric and symmetric dimethylarginine (ADMA and SDMA) and L-arginine plasma concentrations. We recorded Sequential Organ Failure Assessment, Simplified Acute Physiology Score, and Cerebral Performance Category scores. Endpoints were $72 \mathrm{~h}$, intensive care unit, and 30-day mortality. Among 54 enrolled patients [median age: 67 (61-78) years, $48 \%$ male], the initial ADMA levels were significantly elevated in those who died within $72 \mathrm{~h}[0.88(0.64-0.97) \mu \mathrm{mol} / \mathrm{L}$ vs. $0.55(0.45-0.69) \mu \mathrm{mol} / \mathrm{L}, p=0.001]$. Based on receiver operator characteristic analysis $(\mathrm{AUC}=0.723 ; p=0.005)$ of initial ADMA for poor neurological outcome, the best cutoff was determined as $>0.65 \mu \mathrm{mol} / \mathrm{L}$ (sensitivity $=66.7 \%$; specificity $=81.5 \%$ ), while for $72 \mathrm{~h}$ mortality $(\mathrm{AUC}=0.789 ; p=0.001)$ as $>0.81 \mu \mathrm{mol} / \mathrm{L}$ (sensitivity $=71.0 \%$; specificity $=87.5 \%)$. Based on multivariate analysis, initial ADMA $(\mathrm{OR}=1.8$ per $0.1 \mu \mathrm{mol} / \mathrm{L}$ increment; $p=0.002)$ was an independent predictor for $72 \mathrm{~h}$ mortality. Increased initial ADMA predicts $72 \mathrm{~h}$ mortality and poor neurological outcome among unselected cardiac arrest victims.
\end{abstract}

Keywords Cardiopulmonary resuscitation · Post-resuscitation care · Asymmetric dimethylarginine · Prognostication · Cardiac arrest $\cdot$ Mortality

Peter Kenyeres

kenyeres.peter@pte.hu

1 Division of Cardiology, 1st Department of Medicine, Medical School, University of Pécs, Pécs, Hungary

2 Szentagothai Research Centre, University of Pécs, Pécs, Hungary

3 Department of Neurosurgery, Medical School, University of Pécs, Pécs, Hungary

4 Department of Applied Chemistry, University of Debrecen, Debrecen, Hungary

5 Department of Anaesthesiology and Intensive Therapy, Medical School, University of Pécs, Pécs, Hungary

\section{Introduction}

Management of post-resuscitation care, including postcardiac arrest syndrome, ischemic brain injury, myocardial dysfunction, and multiple organ failure (MOF) remains an unmet clinical challenge with high mortality. The 1-year survival rate after out-of-hospital cardiac arrest (OHCA) is around $8 \%$ [1] and 13\% among in-hospital cardiac arrest (IHCA) patients [2]. Early and effective prediction of the mortality, neurological and functional consequences is clinically essential for the medical team to choose the optimal level of treatment, to decide about withdrawal of life-sustaining therapy, to guide goals-of-care conversations with relatives, and to improve the cost-effectiveness of care [3]. Current guidelines recommend a multimodal approach (neurological examination, electrophysiological investigations, neuroimaging, and biomarkers) for prognostication 
of neurological outcome after resuscitation from cardiac arrest [4]. The major limitation of the current algorithm is that it can be applied only to a minority of patients who remain comatose. To improve the quality of prognostication after cardiac arrest, addressing the role of extracerebral causes of death is warranted [5]. Only one-fourth of patients who suffered IHCA die due to neurological injury, while most of them may reach acceptable neurological function but suffer from MOF which may lead to death [6]. A reliable biomarker that can be used in unselected resuscitated patients would provide useful information about the general outcome and survival without focusing only on the neurological status.

Endothelial injury, microcirculatory dysfunction, and coagulopathy developing after resuscitated cardiac arrest are associated with poor outcome [7]. The nitric oxide system plays a crucial role in the regulation of vascular tone and nitric oxide is identified as a mediator of vasodilation under a variety of physiological and pathophysiological conditions, such as hypoxia and ischemia [8]. Enzymatic activities of nitric oxide synthases catalyze the two-step oxidation of L-arginine to nitric oxide and L-citrulline. Methylarginines are described as the main regulators and endogenous inhibitors of nitric oxide synthase catalytic function by competing with L-arginine for binding to the catalytic site of nitric oxide synthase (NG-monomethyl L-arginine and asymmetric dimethylarginine-ADMA) or by binding to the L-arginine membrane uptake carrier (ADMA and symmetric dimethylarginine-SDMA). ADMA has been demonstrated to inhibit nitric oxide formation and increase oxidative stress in vascular endothelial and smooth muscle cells [9].

Previous investigations found a strong association of high plasma ADMA level upon admission and mortality in critically ill patients [10]. Elevated circulating concentrations of $\mathrm{L}$-arginine derivatives have been associated with progression and outcome in a variety of conditions including cardiovascular [11] and cerebrovascular disorders [12-14].

We aimed to explore, for the first time, the alterations of the L-arginine-nitric oxide pathway molecules in the early post-resuscitation care of cardiac arrest survivors, and their distinct association patterns with the prognostic scoring systems, neurological function, and outcome measures such as $72 \mathrm{~h}$, intensive care unit (ICU) and 30-day mortality.

\section{Materials and methods}

\section{Study population}

This is a prospective, single-center observational study conducted from January 2018 to January 2019 in the Intensive care unit of the 1st Department of Medicine, Department of Anaesthesiology and Intensive Care and Department of
Emergency Medicine at the University of Pécs. Our cohort was made up of 54 adult patients [median age: 67 (61-78) years, $48 \%$ male] who suffered IHCA or OHCA, and after successful resuscitation were admitted to the ICU for postresuscitation care. Successful resuscitation was defined as the return of spontaneous circulation (ROSC). 23 patients admitted to the ICU of the 1st Department of Medicine, 18 patients to the Department of Anaesthesiology and Intensive Care, and 13 patients from the Department of Emergency Medicine were enrolled in our cohort. Standard post-resuscitation care was applied for each patient in the ICU without interaction with the research team. Therapeutic hypothermia was not applied during post-resuscitation care, but each patient was kept in normothermia. This report follows the STROBE Statement [15]. The study was approved by the Local Ethics Committee of the University of Pécs (file number: 6941 - PTE 2018.) and has followed the principles outlined in the Declaration of Helsinki for all human investigations. Informed consent for being included in the study was obtained from legal representatives or, in case the patients had regained consciousness, from the patients themselves.

\section{Sample and data collection}

Data collected included patient anamnestic information, comorbidities, the circumstances of cardiopulmonary resuscitation, variables that are necessary for calculating Simplified Acute Physiology Score (SAPS II) and Sequential Organ Failure Assessment (SOFA) severity scores. Mortality by $72 \mathrm{~h}$ after cardiac arrest, mortality occurred in the ICU and 30-day mortality, and the best neurological status was used as outcome measures. Plasma samples were collected within 6,24 , and $72 \mathrm{~h}$ after ROSC to determine the biomarker concentrations by high-performance liquid chromatography. Besides, laboratory and vital parameters were also assessed in the mentioned investigated time points after cardiac arrest. The SAPS II and SOFA scores were calculated according to the worst parameters of the first $24 \mathrm{~h}$ after cardiac arrest. The neurological outcome was measured using cerebral performance category (CPC) score, which consists of a scale of 5 levels: (1) a return to normal cerebral function and normal living, (2) disability but sufficient function for independent activities of daily living, (3) severe disability, limited cognition, inability to carry out independent existence, (4) coma and (5) brain death. CPC scores 1-3 were determined as good and $4-5$ as poor neurological outcome. The best neurological status reached in the ICU was recorded using the CPC scale to avoid false pessimistic neurological classification in patients who regained consciousness after resuscitation and then died due to extracerebral causes with satisfactory neurological status during the follow-up period [16]. 


\section{Biomarkers}

Blood samples were drawn into Vacutainer ${ }^{\circledR}$ EDTA-tubes from resuscitated patients on admission within $6 \mathrm{~h}$ and $24 \pm 3$ and $72 \pm 3 \mathrm{~h}$ after cardiac arrest to determine plasma concentrations of L-arginine, ADMA and SDMA. The samples were centrifuged within $10 \mathrm{~min}$ at $3500 \mathrm{rpm}$ for $15 \mathrm{~min}$. The supernatant was immediately stored in aliquot at $-80^{\circ} \mathrm{C}$ until determining the $\mathrm{L}$-arginine derivative concentrations at the end of the recruitment process. L-arginine, ADMA, and SDMA were measured in the plasma by high-performance liquid chromatography after derivatisation in collaboration with the Department of Applied Chemistry at the University of Debrecen, Hungary $[17,18]$. We calculated the change of the investigated markers from 6 to 24 and 24-72 h. All samples were processed by the same technicians using the same equipment and blinded to all clinical data. The biomarker values were not available for clinical purposes and did not influence therapeutic approaches or the decision-making process.

\section{Statistical analysis}

Statistical analysis of the collected data was evaluated by IBM SPSS Statistics ${ }^{\circledR}$ 27.0. The Kolmogorov-Smirnov test was applied to test for normality of continuous variable distribution. Comparisons of continuous non-normally distributed data between groups were carried out using the Mann-Whitney $U$ test. Student $T$ test was used for analysis of normally distributed continuous data. The continuous variables are reported as medians and interquartile ranges or mean and standard deviation. Correlation analysis was performed calculating Spearman's correlation coefficient (rho). For variables with significant correlation, linear logistic regression analysis was performed, and $R^{2}$ values were reported on the figures. Receiver Operating Characteristic (ROC) analysis and the Area Under the Curve (AUC) were used to determine the most appropriate cutoff values of initial circulating ADMA levels and the investigated endpoints, especially for the evaluation of the $72 \mathrm{~h}$ mortality and neurological outcome based on CPC categories. Univariable binary logistic regression tests were used to evaluate associations between the recorded initial variables and $72 \mathrm{~h}$ mortality with corresponding beta values and $95 \%$ confidence intervals. Variables with $p$ value $\leq 0.05$ in the univariable analysis were included in the multivariable models considering the principle of multicollinearity. Multivariable logistic regression was used to identify factors independently associated with $72 \mathrm{~h}$ mortality. Sample size and power analysis were performed for the overall population using PS program version 3.1.2. For the sample size of $n=54$, patients needed to detect a true difference of $d=0.267$ in initial ADMA for $72 \mathrm{~h}$ mortality with $92 \%$ power, where type I error probability is $\alpha=0.05$. A $p$ value $<0.05$ was considered statistically significant.

\section{Results}

During the 30-day follow-up, $11 \%$ of the patients reached good neurological status (CPC 1-2) and 39\% had severe neurological disability (CPC 3), while half of the patients suffered from coma, vegetative state, or brain death (CPC 4-5). The biomarker levels and their change were also analyzed according to the chosen clinical endpoints. The primary endpoint was the $72 \mathrm{~h}$ mortality, besides, we analyzed ICU mortality, 30-day mortality and neurological outcome (CPC). Figure 1 shows the exact number of survivors during the 30-day follow-up.

\section{2 hour mortality}

Characteristics of the study group made up of 54 successfully resuscitated patients are summarized in Table 1 . Around one-fourth of the patients died within the first 3 days after the ROSC. There was no statistically significant difference between survivors and non-survivors regarding age, gender, cardiac arrest characteristics (e.g., in-hospital or out-of-hospital, length of the cardiopulmonary resuscitation, and first monitored rhythm), suggested etiology of cardiac arrest, vital parameters on enrollment, or

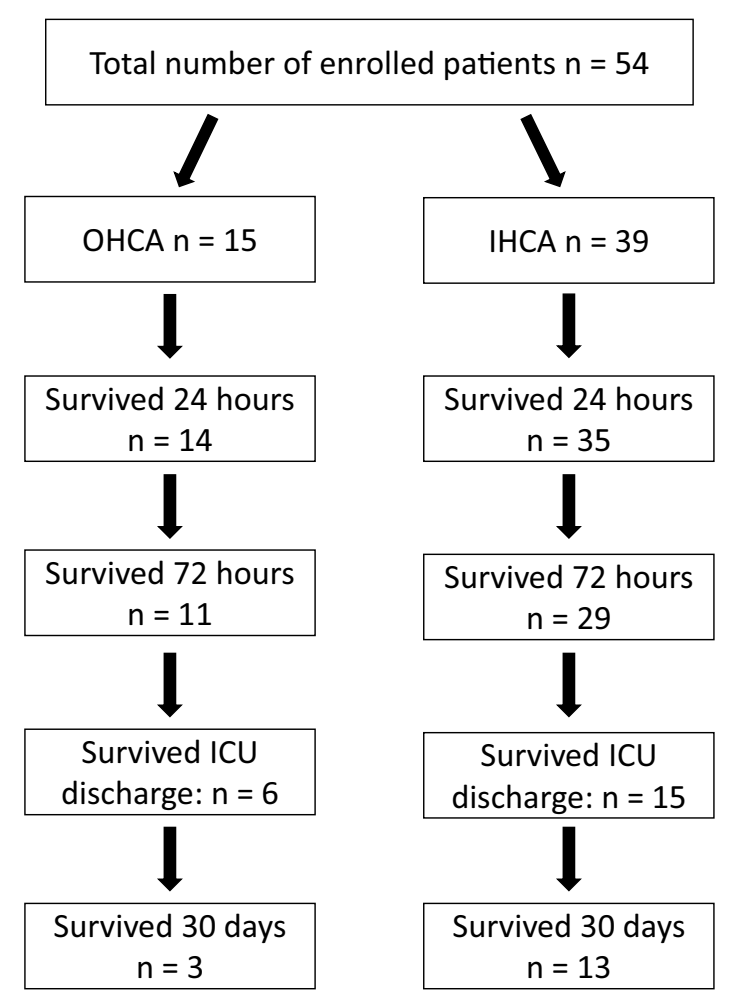

Fig. 1 Study population: Flow-chart about the exact number of survivors (IHCA in-hospital cardiac arrest, $O H C A$ out-of-hospital cardiac arrest, $I C U$ intensive care unit) 
Table 1 Characteristics of the study population according to 72 h mortality

\begin{tabular}{|c|c|c|c|}
\hline & Survivors $(n=40 ; 74 \%)$ & $\begin{array}{l}\text { Non-survivors } \\
(n=14 ; 26 \%)\end{array}$ & $p$ value \\
\hline \multicolumn{4}{|l|}{ Baseline } \\
\hline Age (years) & $66[59-78]$ & $72[63-81]$ & 0.309 \\
\hline Male gender & $21(53 \%)$ & $5(36 \%)$ & 0.279 \\
\hline \multicolumn{4}{|l|}{ Characteristics of the CA and the CPR } \\
\hline Localisation: in-hospital CA & $29(73 \%)$ & $10(71 \%)$ & 0.939 \\
\hline Resuscitation during nightshift or weekend & $28(70 \%)$ & $11(79 \%)$ & 0.538 \\
\hline \multicolumn{4}{|l|}{ First monitored rhythm } \\
\hline Ventricular tachycardia/fibrillation & $9(23 \%)$ & $5(36 \%)$ & 0.332 \\
\hline Pulseless electrical activity & $11(28 \%)$ & $3(21 \%)$ & 0.655 \\
\hline Asystole & $18(45 \%)$ & $5(36 \%)$ & 0.545 \\
\hline Unknown & $2(5 \%)$ & $1(7 \%)$ & 0.762 \\
\hline Time of the resuscitation (min) & $10[5-24]$ & 8 [5-19] & 0.842 \\
\hline Patients required epinephrine & $32(80 \%)$ & $13(93 \%)$ & 0.451 \\
\hline Dose of epinephrine (mg) & $2[1-3]$ & $2[1-3]$ & 0.493 \\
\hline Mechanical ventilation within $6 \mathrm{~h}$ after CA & $37(93 \%)$ & $12(86 \%)$ & 0.451 \\
\hline \multicolumn{4}{|l|}{ Etiology of CA } \\
\hline Ischemic heart disease & $12(30 \%)$ & $5(36 \%)$ & 0.692 \\
\hline Heart failure & $13(33 \%)$ & $3(21 \%)$ & 0.435 \\
\hline Sepsis & $3(8 \%)$ & $2(14 \%)$ & 0.451 \\
\hline Hyperkalaemia & $3(8 \%)$ & $2(14 \%)$ & 0.451 \\
\hline Aspiration & $3(8 \%)$ & 0 & 0.292 \\
\hline Hypothermia & $2(5 \%)$ & 0 & 0.394 \\
\hline Stroke & $1(3 \%)$ & $1(7 \%)$ & 0.429 \\
\hline Pulmonary embolism & $1(3 \%)$ & $1(7 \%)$ & 0.429 \\
\hline Pneumonia & $2(5 \%)$ & 0 & 0.394 \\
\hline Unknown & $14(35 \%)$ & $3(21 \%)$ & 0.347 \\
\hline \multicolumn{4}{|l|}{ Parameters on enrolment } \\
\hline Systolic blood pressure (mmHg) & $115[103-140]$ & $113[95-126]$ & 0.667 \\
\hline Diastolic blood pressure (mmHg) & $61[53-68]$ & $62[57-69]$ & 0.928 \\
\hline Mean arterial pressure $(\mathrm{mmHg})$ & $77[70-91]$ & 76 [71-84] & 0.671 \\
\hline Heart rate (/min) & 78 [65-99] & 94 [79-101] & 0.241 \\
\hline Body temperature $\left({ }^{\circ} \mathrm{C}\right)$ & $36.3 \pm 1.3$ & $36.2 \pm 1.5$ & 0.762 \\
\hline \multicolumn{4}{|l|}{ Comorbidities, previous medical history } \\
\hline Hypertension & $29(73 \%)$ & $10(71 \%)$ & 0.939 \\
\hline Ischemic heart disease & $13(33 \%)$ & $7(50 \%)$ & 0.243 \\
\hline Diabetes mellitus & $19(48 \%)$ & $3(21 \%)$ & 0.088 \\
\hline Heart failure & $14(35 \%)$ & $3(21 \%)$ & 0.347 \\
\hline Permanent atrial fibrillation & $8(20 \%)$ & $2(14 \%)$ & 0.636 \\
\hline Stroke or transient ischemic attack & $8(20 \%)$ & $2(14 \%)$ & 0.636 \\
\hline Carotid artery stenosis & $4(10 \%)$ & $1(7 \%)$ & 0.751 \\
\hline Chronic obstructive pulmonary disease & $8(20 \%)$ & 0 & 0.070 \\
\hline Peripheral artery disease & $5(13 \%)$ & $2(14 \%)$ & 0.864 \\
\hline Previous pulmonary embolism & $2(5 \%)$ & $1(7 \%)$ & 0.763 \\
\hline Previous, cured malignant disease & $5(13 \%)$ & $3(21 \%)$ & 0.418 \\
\hline Active malignant or hematologic disease & $7(18 \%)$ & $2(14 \%)$ & 0.781 \\
\hline \multicolumn{4}{|l|}{ Prognostic scores } \\
\hline SOFA & $10 \pm 3$ & $12 \pm 3$ & 0.267 \\
\hline SAPS II & $70 \pm 16$ & $87 \pm 11$ & $<0.001$ \\
\hline
\end{tabular}

Continuous data are presented as median values with interquartile range [percentiles 25-75] or mean \pm standard deviation, categorical data as the number of subjects and percentages

$C A$ cardiac arrest, $C P R$ cardiopulmonary resuscitation, SOFA Sequential Organ Failure Assessment Score, SAPS II Simplified Acute Physiology Score II, ICU intensive care unit 
comorbidities. $72 \mathrm{~h}$ non-survivors had significantly higher points of SAPS II score.

Table 2 summarizes the absolute plasma levels and the changes of L-arginine, ADMA, and SDMA between the patients who survived the $72 \mathrm{~h}$ after the cardiac arrest or died in this period. Significantly higher initial ADMA levels were observed among patients who died within 3 days. Comparing the initial ADMA levels between the IHCA and OHCA groups, we did not observe significant difference [IHCA: $0.61(0.46-0.85)$ vs. OHCA: $0.64(0.45-0.87), p=0.977]$.

\section{ICU mortality}

A total of 33 patients died in the ICU (by average 6; min. 1 -max. 26 days). Investigating the ICU mortality, none of the L-arginine pathway molecules showed a significant difference between survivors or non-survivors. The ADMA levels tended to remain higher among ICU non-survivors but the difference did not reach significance. The plasma ADMA levels of ICU non-survivors decreased from 6 to $24 \mathrm{~h}$, while the values of the surviving group elevated by $24 \mathrm{~h}[-0.08$ ( -0.16 to 0.05$) \mu \mathrm{mol} / \mathrm{L}$ vs. 0.07 ( -0.04 to 0.11$) \mu \mathrm{mol} / \mathrm{L}$, $p=0.024]$ (Suppl.-Table 1). Subgroup analysis of IHCA patients revealed significantly decreased $6 \mathrm{~h}$ L-arginine/ ADMA ratio in patients who died in the ICU (Suppl.-Fig. 1).

\section{0-day mortality}

$70 \%$ of the patients died within 30 days after cardiac arrest. Analyzing the kinetics of the markers according to 30-day mortality outcome, an opposite change was observed in ADMA level from 6 to $24 \mathrm{~h}$ between survivors and non-survivors similarly to the observation according to ICU mortality $[-0.08(-0.16$ to 0.06$)$ in non-survivors vs. $0.07(-0.03$ to 0.11 ) in survivors, $p=0.028$ ] (Suppl.-Table 2). In contrast,
L-arginine, SDMA levels, or their change showed no significant difference in any of the clinical endpoints at any investigated time point. The L-arginine/ADMA ratio slightly elevated up to 72 post-cardiac arrest hours in the total population regardless of the mortality ( $6 \mathrm{~h}: 66.04 \pm 4.33 ; 24 \mathrm{~h}$ : $80.04 \pm 5.35 ; 72$ h: $99.99 \pm 7.13 ; p<0.05)$ (Suppl.-Fig. 2).

\section{Prognostic scores and biomarkers}

The SAPS II and SOFA score had significant but moderate prognostic value for ICU mortality based on ROC analysis [SOFA AUC: $0.695(0.537-0.853) p=0.020$; SAPS II AUC: 0.747 (0.602-0.891), $p=0.003$ ]. Neither SAPS II nor SOFA score showed significant difference between IHCA and OHCA subgroups. The statistical analysis revealed a significant positive correlation between the initial ADMA levels and the SAPS II score ( $\mathrm{rho}=0.393$, $R^{2}=0.178, p=0.002$ ) (Suppl.-Fig. 3). The L-arginine levels per se did not show significant correlation with the investigated scores or parameters. Figure 2 demonstrates the analysis of the three parameters (SOFA, SAPS II, and initial ADMA) for $72 \mathrm{~h}$ mortality in a combined ROC curve. The results showed that the AUC of SAPS II and initial ADMA were comparable reflecting similar sensitivity and specificity in prediction of $72 \mathrm{~h}$ mortality, in contrast SOFA provided poor prognostic information for mortality [SAPS II AUC: $0.817(0.688-0.946), p<0.001$; ADMA AUC: $0.789(0.628-0.950), p=0.001$; SOFA AUC: 0.608 (0.433-0.783), $p=0.232$ ].

\section{Neurological outcome}

The initial ADMA levels were significantly elevated among patients with poor neurological outcome (CPC 4-5)
Table 2 L-Arginine pathway molecules and their change according to the $72 \mathrm{~h}$ mortality

\begin{tabular}{lccc}
\hline & Survivors $(n=40 ; 74 \%)$ & Non-survivors $(n=14 ; 26 \%)$ & $p$ value \\
\hline Biomarker plasma levels within $6 \mathrm{~h}$ after CA & & \\
L-arginine $(\mu \mathrm{mol} / \mathrm{L})$ & $33.45[27.84-46.96]$ & $46.16[27.89-72.44]$ & 0.079 \\
ADMA $(\mu \mathrm{mol} / \mathrm{L})$ & $0.55[0.45-0.69]$ & $0.88[0.64-0.97]$ & 0.001 \\
SDMA $(\mu \mathrm{mol} / \mathrm{L})$ & $0.93[0.65-1.60]$ & $0.93[0.76-1.29]$ & 0.969 \\
Biomarker plasma levels 24 h after CA & $38.95[31.26-60.56]$ & $45.62[17.64-70.11]$ & 0.910 \\
L-arginine $(\mu \mathrm{mol} / \mathrm{L})$ & $0.54[0.45-0.78]$ & $0.78[0.51-1.05]$ & 0.145 \\
ADMA $(\mu \mathrm{mol} / \mathrm{L})$ & $1.03[0.75-1.98]$ & $1.32[0.88-2.28]$ & 0.515 \\
SDMA $(\mu \mathrm{mol} / \mathrm{L})$ & $5.16[-4.48$ to 23.37$]$ & $-5.21[-25.32$ to 21.38$]$ & 0.234 \\
Change in biomarker plasma levels from 6 to $24 \mathrm{~h}$ after CA & & 0.079 \\
$\Delta \mathrm{L}-$ arginine $(24-6 \mathrm{~h})(\mu \mathrm{mol} / \mathrm{L})$ & $5.03[-0.08$ to 0.10$]$ & $-0.12[-0.20$ to 0.02$]$ & 0.713 \\
$\Delta$ ADMA $(24-6 \mathrm{~h})(\mu \mathrm{mol} / \mathrm{L})$ & $0.17[-0.02$ to 0.42$]$ & $0.22[0.02-0.42]$ & \\
$\Delta$ SDMA $(24-6 \mathrm{~h})(\mu \mathrm{mol} / \mathrm{L})$ &
\end{tabular}

Data are presented as median values with interquartile range [percentiles 25-75]

$A D M A$ asymmetric dimethylarginine, $C A$ cardiac arrest, $S D M A$ symmetric dimethylarginine 
Fig. 2 ROC Curve of initial ADMA, SOFA, and SAPS II for 72-day mortality. SAPS II AUC: 0.817 [0.688-0.946], $p<0.001$; ADMA AUC: 0.789 [0.628-0.950], $p=0.001 ;$ SOFA AUC: 0.608 [0.433-0.783], $p=0.232(A D M A$ asymmetric dimethylarginine, $A U C$ Area Under the Curve, SAPS Simplified Acute Physiology Score, SOFA Sequential Organ Failure Assessment, ROC Receiver Operating Characteristic)
ROC Curve of initial ADMA, SOFA and SAPS II for 72-hour mortality

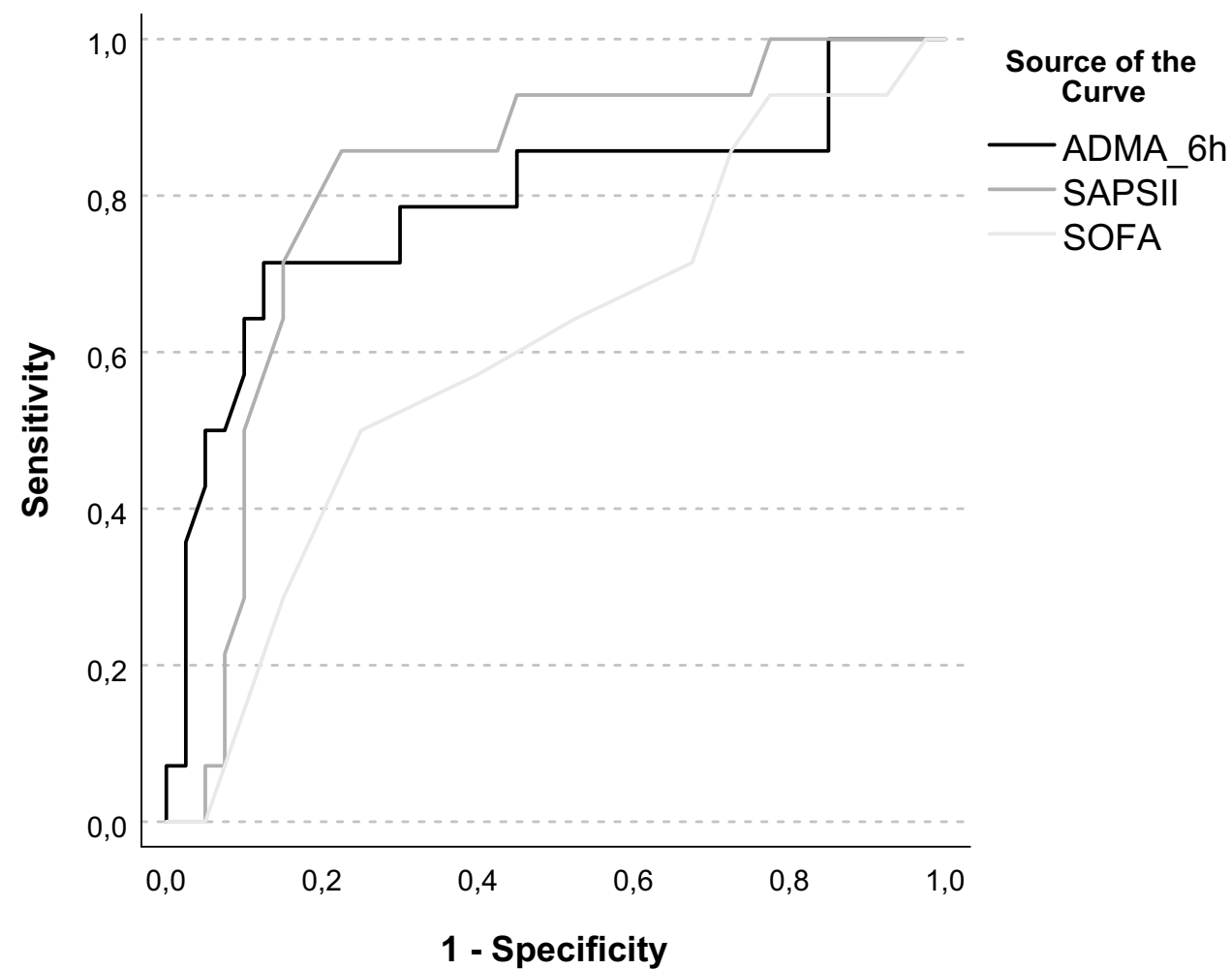

Fig. 3 ADMA and neurological outcome (max. CPC). (ADMA asymmetric dimethylarginine, $C P C$ cerebral performance category)

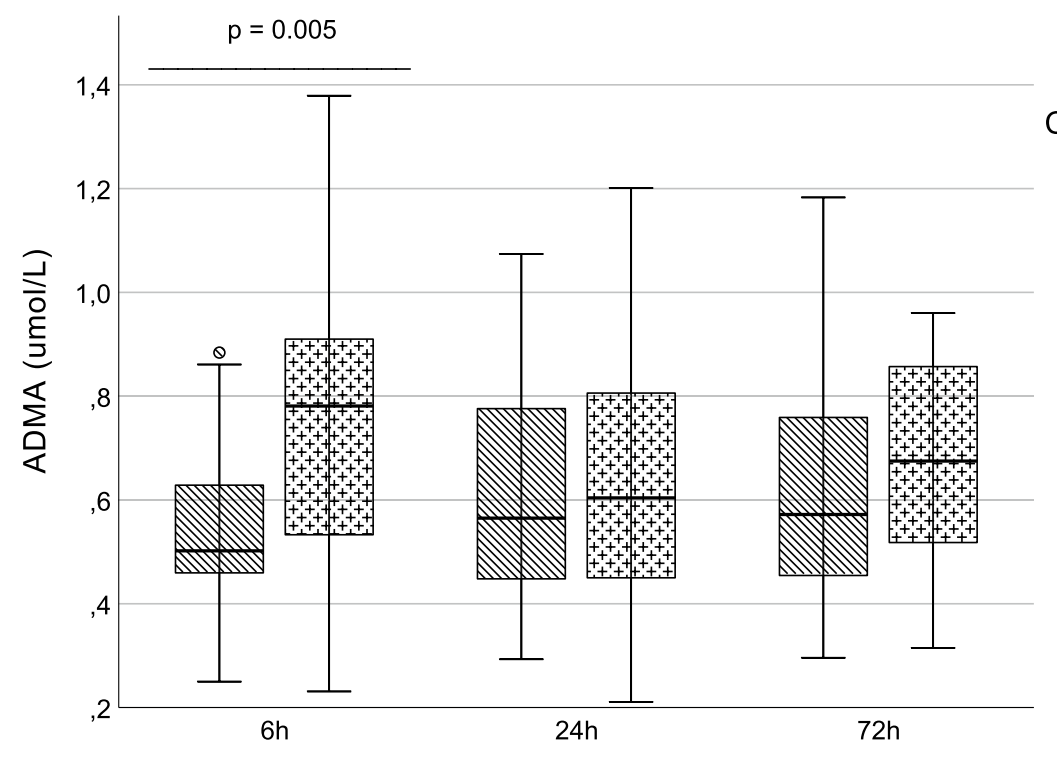

Cerebral

Performance Category (CPC)

$\mathbb{N C P C} 1-3$

$+\mathrm{FPC} 4-5$
(Fig. 3). ROC analysis of initial ADMA for prediction of a coma, vegetative state, or brain death (CPC 4-5) showed an AUC of 0.723 (95\% CI 0.574-0.871; $p=0.005)$. Based on the ROC analysis, the best cutoff for poor neurological outcome (CPC 4-5) was determined as $>0.65 \mu \mathrm{mol} / \mathrm{L}$ (sensitivity: $66.7 \%$; specificity: $81.5 \%$ ).

\section{Independent prediction of $72 \mathrm{~h}$ mortality}

Based on ROC analysis, initial ADMA level was found to be a predictor of $72 \mathrm{~h}$ mortality (Fig. 2) with a best cutoff value of $>0.81 \mu \mathrm{mol} / \mathrm{L}$ (sensitivity: $71.0 \%$; specificity: $87.5 \%$ ). Univariable logistic regression analyses including each variable assessed within $6 \mathrm{~h}$ after cardiac arrest 
identified initial ADMA, serum bicarbonate, and lactate levels as significant markers for $72 \mathrm{~h}$ mortality. Multivariable analysis revealed that initial ADMA (OR: 1.8 per $0.1 \mu \mathrm{mol} / \mathrm{L}$ increase in ADMA; 95\% CI 1.252-2.611; $p=0.002)$ is an independent predictor for $72 \mathrm{~h}$ outcome after cardiac arrest (Table 3).

\section{Discussion}

To the best of our knowledge, the L-arginine pathway molecules and their change in the early post-resuscitation phase have not yet been evaluated in unselected cardiac arrest patients. Here, we investigated the prognostic value of L-arginine, ADMA, SDMA plasma levels, and kinetics in combination with other conventionally used laboratory parameters among a general, unselected population of cardiac arrest survivors including IHCA and OHCA patients. The major result of our study was the observation that initial ADMA level measured within $6 \mathrm{~h}$ after cardiac arrest was an independent predictor of short-term mortality and poor neurological outcome. While the $6 \mathrm{~h}$ ADMA levels had unequivocal prognostic value for $72 \mathrm{~h}$ mortality, the levels measured at either 24 and $72 \mathrm{~h}$ or their change did not associate with any of the investigated endpoints. Neither ICU nor 30-day mortality was predicted by any of the L-arginine pathway molecules. It was described previously that cardiovascular failure and hemodynamical instability are responsible for early death within 3 days after cardiac arrest, while later death is mainly related to neuronal injury due to severe hypoxic-ischemic encephalopathy and the subsequent withdrawal of life-sustaining therapy [19]. The different pathophysiological backgrounds of early and later post-resuscitation death may explain that ADMA, as a prognostic marker associated with the severity and mortality of many cardiovascular diseases, may also be promising for predicting mortality in the early phase of post-resuscitation care [20]. Accordingly, we could not prove the prognostic value of SDMA and L-arginine for mortality in the post-resuscitation phase.

A most recent review about the metabolism of ADMA in hypoxia summarizes the results of both animal and human studies concerning the metabolites of $\mathrm{L}$-arginine pathway in various hypoxic conditions [21]. They mention more studies where ADMA levels were elevated in hypoxia, while SDMA levels did not show significant elevation. One of them found continuous increase of ADMA but not of SDMA in chronicintermittent hypobaric hypoxia [22]. Others observed higher ADMA serum concentrations in patients with obstructive sleep apnea syndrome [23]. Furthermore, the L-arginine pathway molecules have been suggested as prognostic markers for acute exacerbation of chronic obstructive pulmonary disease [24].

Previous publications investigated the L-arginine pathway molecules in ischemic stroke [13, 25]. Plasma ADMA levels were higher in acute ischemic stroke patients compared to the control group and stroke outcome was worse in patients with increased ADMA levels than those with stable ADMA levels. They explained the observation with the mechanism that increased ADMA levels inhibit the production of endothelial nitric oxide (which plays a crucial role as an endogenous regulator of vasodilation in cerebral arterioles) consequently reducing the cerebral perfusion [25]. Other investigations also confirmed that ADMA increases the vascular tone in cerebral blood vessels and leads to cerebral hypoperfusion [26]. Molnar et al. observed that metabolites

Table 3. Univariable (a) and multivariable (b) regression analysis for $72 \mathrm{~h}$ mortality

a. Univariable logistic regression analysis for $72 \mathrm{~h}$ mortality

\begin{tabular}{|c|c|c|c|}
\hline Variable & & Odds ratio-Exp(B) (lower CI-upper CI) & $p$ \\
\hline ADMA $6 \mathrm{~h}$ (per $0.1 \mu \mathrm{mol} / \mathrm{L}$ increase) & & $1.81(1.25-2.61)$ & 0.002 \\
\hline $\mathrm{HCO}_{3}^{-} 6 \mathrm{~h}$ & & $0.89(0.79-0.99)$ & 0.034 \\
\hline Lactate $6 \mathrm{~h}$ & & $1.26(1.06-1.49)$ & 0.008 \\
\hline \multicolumn{4}{|c|}{ b. Binary logistic regression analysis for $72 \mathrm{~h}$ mortality } \\
\hline $\begin{array}{l}\text { Model 1-Binary Logistic Regression- } \\
\text { Enter }\end{array}$ & $B$ & Odds ratio-Exp(B) (lower CI-upper CI) & $p$ \\
\hline ADMA $6 \mathrm{~h}$ (per $0.1 \mu \mathrm{mol} / \mathrm{L}$ increase) & 0.573 & $1.77(1.23-2.56)$ & 0.002 \\
\hline $\mathrm{HCO}_{3}^{-} 6 \mathrm{~h}$ & -0.132 & $0.88(0.77-1.00)$ & 0.054 \\
\hline Model 2-Binary Logistic Regression-Enter & $\mathrm{B}$ & Odds ratio-Exp(B) (lower CI-upper CI) & $\mathrm{p}$ \\
\hline ADMA $6 \mathrm{~h}$ (per $0.1 \mu \mathrm{mol} / \mathrm{L}$ increase) & 0.488 & $1.63(1.14-2.33)$ & 0.008 \\
\hline Lactate $6 \mathrm{~h}$ & 0.189 & $1.21(0.99-1.48)$ & 0.065 \\
\hline
\end{tabular}

ADMA asymmetric dimethylarginine, $C I$ confidence interval 
of the L-arginine pathway were elevated in the very acute phase of ischemic stroke indicating a more pronounced endothelial dysfunction compared with asymptomatic significant carotid stenosis or healthy subjects. They suggested that the elevated initial ADMA levels could be linked to the pathogenesis of endothelial cell dysfunction or could be the consequence of oxidative stress [13].

We observed a decrease from higher initial levels of ADMA levels up to $24 \mathrm{~h}$ among patients who died within 30 days after cardiac arrest, while ADMA levels slightly elevated in survivors. The discrepancy between the initial ADMA levels of non-survivors and survivors disappeared by 24 post-cardiac arrest hours. ADMA per se might contribute to brain injury by either reducing cerebral blood flow and facilitating excitotoxic neuronal death or contributing to the activation of the thrombo-inflammatory cascade [12]. Excessively high initial ADMA may adversely affect cerebral perfusion after cardiac arrest, leading in the short term to exacerbation of hypoxic-ischemic injury following resuscitation and early death in the post-resuscitation phase. On the other hand, elevated initial ADMA levels might indicate a more severe hypoxic insult or pre-existing endothelial dysfunction as discussed in the publication of Molnar et al. mentioned above. In the literature, only a few studies are investigating these marker levels in acute hypoxic conditions in humans. In healthy male volunteers, the mean plasma nitric oxide concentration was elevated after acute hypoxic exposure, which was associated with a reduction in plasma ADMA level leading to elevated plasma nitric oxide concentrations [27]. This finding suggests that the decrease in ADMA concentration observed on the first day after cardiac arrest in our more severe group of patients who died within 30 days may be an adaptive mechanism presumably counteracting cerebral hypoperfusion. Although it is important to note that the concentration of nitric oxide was not determined in our present study.

We observed a continuous increase of L-arginine/ADMA ratio up to 72 post-cardiac arrest hours in the total study population and found a significantly decreased L-arginine/ ADMA ratio at 6 post-cardiac arrest hours in patients who died in the ICU after IHCA. These findings are consistent with the observation of Molnar et al., who suggested that a temporary increase of L-arginine along with a decrease of ADMA could be a protective mechanism after ischemic stroke [13]. Nitric oxide, a pleiotropic molecule, has several intracellular effects leading to vasorelaxation, endothelial regeneration, inhibition of leukocyte chemotaxis, and platelet adhesion [28]. In turn, lack of L-arginine, the source of nitric oxide, could lead to oxidative stress induced by hypoxic insults such as cardiac arrest, thus it may explain the reduced initial L-arginine/ADMA ratio in IHCA nonsurvivors. A most recent prospective observational study found that a higher arginine and lower arginine/ADMA ratio measured within $24 \mathrm{~h}$ after OHCA were independently associated with 90-day mortality [29]. Similarly to their findings, we could detect significantly elevated L-arginine/ADMA ratio in survivors in the IHCA group. They did not find significant difference between survivors and non-survivors regarding ADMA levels. Despite their findings, L-arginine was not a prognosticator of death in our population. Moreover, this marker did not show difference between survivors and non-survivors for none of the investigated endpoints. However, their population was exclusively made up of patients who suffered OHCA, while we enrolled mostly IHCA patients. Importantly, the pathophysiology and the most common conditions leading to death during postresuscitation care could be different. Two-thirds of OHCA patients die due to brain injury in ICU, while MOF is the main cause of mortality after IHCA [6].

In this study, we recorded the best CPC reached in the ICU. It is generally observed, that despite patients reach acceptable neurological function during their ICU stay, they may later die as the consequence of MOF, especially after IHCA [6]. Considering this, we felt it confusing to categorize patients emerging from a comatose state into the unfavorable CPC group based on late-developing MOF. Therefore, the pure neurological outcome (regaining the consciousness) and the overall outcome (ICU or total mortality) should be separately analyzed. Therefore, the best achieved CPC score was recorded during the ICU stay similar to the study of Fabio Silvio Taccone and colleagues [16]. Importantly, eight patients (14.8\% of the total population) in the CPC 1-3 group died due to non-neurological reasons in the ICU despite acceptable neurological status (death after awakening). All these eight patients had sepsis and failure of two or more organ systems.

The reliability of SOFA and SAPS II scores for prognostication in cardiac arrest patients remains unclear [30]. In our population, these scores had moderate prognostic value and we confirmed their association with L-arginine pathway molecules. Previous research findings revealed correlations among ADMA levels, L-arginine/ADMA ratio and microvascular reactivity, the extent of organ failure, and mortality in patients with sepsis [31]. In critically ill patients, ADMA correlated with SOFA score [10]. These observations are consistent with our results. Based on our findings, the prognostic accuracy of SAPS II (a time-consuming assessment method) and initial ADMA for $72 \mathrm{~h}$ mortality after ROSC were comparable. We conclude that early determination of initial ADMA after ROSC may be as effective and accurate as SAPS II in prediction of the early post-CPR mortality.

The strength of our study is its prospective nature using serial sampling in the first 3 days after cardiac arrest to evaluate the kinetics and changes of the L-arginine pathway molecules. Our study population was made up of unselected resuscitated patients including in- and out-of-hospital 
cardiac arrest survivors of three different intensive care units allowing us to explore reliable predictive markers regardless of the circumstances and etiology of cardiac arrest and covering the widest range of resuscitated patients with different comorbidities and etiology. While most studies evaluated prognostication of OHCA patients, here, we were able to find potential prognostic markers in IHCA patients and markers which might be also used in both groups. The best neurological status reached in the intensive care unit was recorded to avoid false pessimistic neurological estimation of patients (especially after in-hospital cardiac arrest) reaching acceptable neurological status but dying as the consequence of hemodynamic dysfunction or MOF.

The limitation of our study is the low total number of enrolled patients. Concerning the high mortality rate, we could not collect enough data for long-term analysis. Further research is warranted to explore the prognostic value of multiple markers (including the L-arginine pathway molecules) for long-term outcome after cardiac arrest. Probably, the no-flow time (i.e., the interval between collapse and start of CPR) would be more valuable information compared to the length of the CPR until ROSC. However, the exact time of the collapse was not known in the majority of IHCA and some OHCA patients, thus it prevented us from reporting the accurate no-flow time in this cohort. To satisfy the proportional odds assumption, we reclassified the CPC as follows: CPC 4-5 (vegetative state or death) as poor neurological outcome, and CPC 1-3 as good/moderate neurological outcome, because of the low number of individuals with really good outcome (CPC 1-2) until 30-day follow-up. The reason of the extension of the acceptable neurological outcome categories with CPC 3 was the potential for a later neurological improvement of some patients during rehabilitation. The study was conducted in a single-center, so local treatment strategies and guidelines could limit the generalisability of our findings.

\section{Conclusions}

Here, we investigated for the first time the prognostic value and the kinetics of the L-arginine-pathway molecules during the early phase of successful cardiopulmonary resuscitation. Our results suggest that initial circulating ADMA level may indicate more severe hypoxic insult and can predict $72 \mathrm{~h}$ mortality among cardiac arrest victims.

Supplementary Information The online version contains supplementary material available at https://doi.org/10.1007/s11739-021-02767-z.

Author contributions PK, ZM, PC, KT, RH, and BC designed the study protocol. ZM, JR, PK, and BC enrolled the patients, collected the data and samples. LN contributed important reagents and performed the measurement. BC, TM, and BS analyzed the data. BC and TM interpreted the results, wrote the paper, and all the authors reviewed the paper prior to submission.

Funding Open access funding provided by University of Pécs. This work was funded by GINOP 2.3.2.-15-2016-00048 and by NKFIH in Hungary, within the framework of the 2020-4.1.1-TKP2020 1st thematic program of the University of Pécs. This work was supported by EFOP-3.6.3- VEKOP-16-2017-00009 at the University of Pécs.

Availability of data and material All data relevant to the study are included in the article or uploaded as supplementary material. No additional data are available due to data protection requirements.

\section{Declarations}

Conflict of interest The authors have no financial involvement with any organization or entity with a financial interest in or financial conflict with the subject matter or materials discussed in the manuscript apart from those disclosed. The authors declare no competing interests.

Human and animal rights statement and ethics approval The study was approved by the Local Ethics Committee of the University of Pécs (file number: 6941-PTE 2018.) and has followed the principles outlined in the Declaration of Helsinki for all human investigations.

Informed consent Informed consent for being included in the study was obtained from legal representatives or, in case, the patients had regained consciousness, from the patients themselves.

Open Access This article is licensed under a Creative Commons Attribution 4.0 International License, which permits use, sharing, adaptation, distribution and reproduction in any medium or format, as long as you give appropriate credit to the original author(s) and the source, provide a link to the Creative Commons licence, and indicate if changes were made. The images or other third party material in this article are included in the article's Creative Commons licence, unless indicated otherwise in a credit line to the material. If material is not included in the article's Creative Commons licence and your intended use is not permitted by statutory regulation or exceeds the permitted use, you will need to obtain permission directly from the copyright holder. To view a copy of this licence, visit http://creativecommons.org/licenses/by/4.0/.

\section{References}

1. Yan S, Gan Y, Jiang N et al (2020) The global survival rate among adult out-of-hospital cardiac arrest patients who received cardiopulmonary resuscitation: a systematic review and meta-analysis. Crit Care 24(1):61

2. Schluep M, Gravesteijn BY, Stolker RJ, Endeman H, Hoeks SE (2018) One-year survival after in-hospital cardiac arrest: a systematic review and meta-analysis. Resuscitation 132:90-100

3. Elmer J, Torres C, Aufderheide TP et al (2016) Association of early withdrawal of life-sustaining therapy for perceived neurological prognosis with mortality after cardiac arrest. Resuscitation 102:127-135

4. Panchal AR, Bartos JA, Cabañas JG et al (2020) Part 3: adult basic and advanced life support: 2020 American heart association guidelines for cardiopulmonary resuscitation and emergency cardiovascular care. Circulation 142(16_suppl_2):S366-s468 
5. Sandroni C, Grippo A, Nolan JP (2020) ERC-ESICM guidelines for prognostication after cardiac arrest: time for an update. Intensive Care Med 46(10):1901-1903

6. Laver S, Farrow C, Turner D, Nolan J (2004) Mode of death after admission to an intensive care unit following cardiac arrest. Intensive Care Med 30(11):2126-2128

7. Grundmann S, Fink K, Rabadzhieva L et al (2012) Perturbation of the endothelial glycocalyx in post cardiac arrest syndrome. Resuscitation 83(6):715-720

8. Böger R, Hannemann J (2020) Dual role of the L-arginine-ADMANO pathway in systemic hypoxic vasodilation and pulmonary hypoxic vasoconstriction. Pulm Circ 10(2):2045894020918850

9. Tran CT, Leiper JM, Vallance P (2003) The DDAH/ADMA/NOS pathway. Atheroscler Suppl 4(4):33-40

10. Koch A, Weiskirchen R, Kunze J et al (2013) Elevated asymmetric dimethylarginine levels predict short- and long-term mortality risk in critically ill patients. J Crit Care 28(6):947-953

11. Szabo Z, Bartha E, Nagy L, Molnar T (2020) Increased symmetric dimethylarginine, but not asymmetric dimethylarginine, concentrations are associated with transient myocardial ischemia and predict outcome. J Int Med Res 48(6):300060520920439

12. Molnar T, Pusch G, Nagy L, Keki S, Berki T, Illes Z (2016) Correlation of the L-arginine pathway with thrombo-inflammation may contribute to the outcome of acute ischemic stroke. J Stroke Cerebrovasc Dis 25(8):2055-2060

13. Molnar T, Pusch G, Papp V et al (2014) The L-arginine pathway in acute ischemic stroke and severe carotid stenosis: temporal profiles and association with biomarkers and outcome. J Stroke Cerebrovasc Dis 23(8):2206-2214

14. Csecsei P, Várnai R, Nagy L et al (2019) L-arginine pathway metabolites can discriminate paroxysmal from permanent atrial fibrillation in acute ischemic stroke. Ideggyogy Sz 72(3-4):79-88

15. Vandenbroucke JP, Von Elm E, Altman DG et al (2007) Strengthening the reporting of observational studies in epidemiology (STROBE): explanation and elaboration. Ann Intern Med 147(8):W163-194

16. Taccone FS, Horn J, Storm C et al (2019) Death after awakening from post-anoxic coma: the "Best CPC" project. Crit Care 23(1): 107

17. Nonaka S, Tsunoda M, Imai K, Funatsu T (2005) High-performance liquid chromatographic assay of $\mathrm{N}(\mathrm{G})$-monomethyl-L-arginine, $\mathrm{N}(\mathrm{G}), \mathrm{N}(\mathrm{G})$-dimethyl-L-arginine, and $\mathrm{N}(\mathrm{G}), \mathrm{N}(\mathrm{G})^{\text {'-dime- }}$ thyl-L-arginine using 4-fluoro-7-nitro-2, 1,3-benzoxadiazole as a fluorescent reagent. J Chromatogr A 1066(1-2):41-45

18. Molnár-Perl I, Vasanits A (1999) Stability and characteristics of the o-phthaldialdehyde/3-mercaptopropionic acid and o-phthaldialdehyde/ $N$-acetyl-1-cysteine reagents and their amino acid derivatives measured by high-performance liquid chromatography. Presented at the 22nd international symposium on highperformance liquid phase separations and related techniques, St. Louis, MO, 3-8 May 1998. J Chromatogr A 835(1):73-91
19. Dragancea I, Rundgren M, Englund E, Friberg H, Cronberg T (2013) The influence of induced hypothermia and delayed prognostication on the mode of death after cardiac arrest. Resuscitation 84(3):337-342

20. Böger RH, Sullivan LM, Schwedhelm E et al (2009) Plasma asymmetric dimethylarginine and incidence of cardiovascular disease and death in the community. Circulation 119(12):1592-1600

21. Hannemann J, Zummack J, Hillig J, Böger R (2020) Metabolism of asymmetric dimethylarginine in hypoxia: from bench to bedside. Pulm Circ 10(2):2045894020918846

22. Lüneburg N, Siques P, Brito J et al (2017) Long-term intermittent exposure to high altitude elevates asymmetric dimethylarginine in first exposed young adults. High Alt Med Biol 18(3):226-233

23. İn E, Özdemir C, Kaman D, Sökücü SN (2015) Heat shock proteins, l-arginine, and asymmetric dimethylarginine levels in patients with obstructive sleep apnea syndrome. Arch Bronconeumol 51(11):544-550

24. Ruzsics I, Nagy L, Keki S et al (2016) L-Arginine pathway in COPD patients with acute exacerbation: a new potential biomarker. COPD 13(2):139-145

25. Worthmann H, Chen S, Martens-Lobenhoffer J et al (2011) High plasma dimethylarginine levels are associated with adverse clinical outcome after stroke. J Atheroscler Thromb 18(9):753-761

26. Kielstein JT, Donnerstag F, Gasper S et al (2006) ADMA increases arterial stiffness and decreases cerebral blood flow in humans. Stroke 37(8):2024-2029

27. Lambden S, Martin D, Vanezis K et al (2016) Hypoxia causes increased monocyte nitric oxide synthesis which is mediated by changes in dimethylarginine dimethylaminohydrolase 2 expression in animal and human models of normobaric hypoxia. Nitric Oxide 58:59-66

28. Dias RG, Negrão CE, Krieger MH (2011) Nitric oxide and the cardiovascular system: cell activation, vascular reactivity and genetic variant. Arq Bras Cardiol 96(1):68-75

29. Keller A, Becker C, Nienhaus K et al (2020) Arginine and arginine/ADMA ratio predict 90-day mortality in patients with out-ofhospital cardiac arrest-results from the prospective observational COMMUNICATE Trial. J Clin Med 9(12):3815

30. Choi JY, Jang JH, Lim YS et al (2018) Performance on the APACHE II, SAPS II, SOFA and the OHCA score of post-cardiac arrest patients treated with therapeutic hypothermia. PLoS ONE 13(5): $\mathrm{e} 0196197$

31. Davis JS, Darcy CJ, Yeo TW et al (2011) Asymmetric dimethylarginine, endothelial nitric oxide bioavailability and mortality in sepsis. PLoS ONE 6(2):e17260

Publisher's Note Springer Nature remains neutral with regard to jurisdictional claims in published maps and institutional affiliations. 